\title{
Long-term survival and prognostic factors for unfavorable outcome in patients with systemic sclerosis. A prospective single-center study
}

\author{
Alexandra Daniela Radu ${ }^{1,2}$, Ana Maria Gheorghiiu ${ }^{1,2}$, Raida Oneata ${ }^{1,2}$, Alina Soare ${ }^{1,2}$, \\ Ruxandra Dobrota ${ }^{1,2}$, Stefania Magda ${ }^{2,3}$, Tudor Constantinescu ${ }^{2,4}$, Ruxandra Jurcut ${ }^{2,5}$, \\ Roxana Sfrent-Cornateanu ${ }^{2,6}$, Mihai Bojinca ${ }^{1,2}$, Victor Stoica ${ }^{1,2}$, Carina Mihai ${ }^{1,2}$ \\ ${ }^{1}$ Internal Medicine and Rheumatology, "Dr. I. Cantacuzino" Clinical Hospital, Bucharest, Romania \\ 2"Carol Davila" University of Medicine and Pharmacy, Bucharest, Romania \\ 3Emergency University Hospital, Bucharest, Romania \\ 4"Prof. Dr. Marius Nasta" Institute of Pneumology, Bucharest, Romania \\ 5"Prof. Dr. C.C. Iliescu" Institute for Cardiovascular Diseases, Bucharest, Romania \\ ${ }^{6}$ Physiopathology and Immunology Department, Bucharest, Romania
}

\begin{abstract}
Background. Systemic sclerosis (SSc) is a complex chronic autoimmune disease, with an unpredictable evolution and high morbidity and mortality rates.

Objective. Evaluation of long-term survival and identification of prognostic factors in patients with systemic sclerosis.

Methods. All patients with SSc of the EUSTAR100 center, having at least one visit between 2004 and 2016, were included. Data were analyzed for survival, cause of death, as well as for the following events defining disease worsening: increase in modified Rodnan score (mRSS) with at least $25 \%$ and 5 points (compared to baseline visit), decrease with at least 10\% (compared to baseline) of predicted forced vital capacity (FVC) and predicted diffusing capacity of the lungs for carbon monoxide (DLCO), and presence of new digital ulcers (DUs). Logistic regression (LR), Cox proportional hazards regression and Kaplan-Meier survival curves were used in univariate and multivariate analysis to study survival and identify prognostic factors.

Results. 137 patients were included in the study (89.1\% females, mean age \pm SD $56.7 \pm 12.6$ years, disease duration $9.7 \pm 7.1$ years), with a follow-up duration of up to 19 years. 96 patients had at least one follow-up visit and 66 (not including patients who died earlier than 2 years after the first presentation) had follow-up data at 2 years ( \pm 6 months) after the first visit in the clinic. There were 19 reported deaths $(13.9 \%), 11$ attributed to SSc (of whom 8 were due to lung involvement). Risk factors for death were diffuse cutaneous subset and mRSS $>14$ at baseline (identified by LR adjusted for age and sex), male sex and proteinuria (Cox analysis).

While in over half of the patients FVC and mRSS were stable or improved ( $86 \%$ and $96 \%$ respectively), and no new DUs occurred (64\%), $52 \%$ of the patients presented significant worsening of DLCO during the entire followup. Risk factors for DLCO worsening at 2 years, by LR adjusted for sex and age, were male sex and diffuse cutaneous subset, while Cox analysis identified only male sex. The only risk factor identified for appearance of new DUs was the history of DUs at the first presentation.

Conclusions. SSc often presents an unfavorable disease course, particularly due to lung involvement. Risk factors for disease worsening were male sex, diffuse cutaneous subset, and mRSS $>14$ at baseline. SSc-related deaths were mainly due to lung involvement, thus underlining the necessity of identifying predictive factors for lung function deterioration at the first presentation.
\end{abstract}

Keywords: systemic sclerosis, prognostic factors, survival

\section{INTRODUCTION AND OBJECTIVES}

Systemic sclerosis (SSc) is a chronic systemic autoimmune disease with a variable course. SSc has the highest mortality rate of all connective tissue diseases, death occurring most frequently due to interstitial lung disease (ILD) (1). Other leading SSc-re- lated death causes are severe gastrointestinal involvement (leading to malnutrition), heart involvement and scleroderma renal crisis $(2,3)$. Studies report important early mortality in SSc (4). Therefore, identifying prognostic factors at the first presentation of SSc patients in a specialized center is important for optimal management (1). This can be 
achieved by observing a large number of patients over a long period of time. This study is aiming to evaluate long-term survival and to identify prognostic factors in a Romanian single-center cohort of patients with systemic sclerosis.

\section{PATIENTS AND METHODS}

All patients diagnosed with SSc by expert opinion, who attended the EUSTAR center 100 between Jan 2004 and March 2016, were included in this study. An extensive clinical, imagistic, functional and laboratory investigation was performed in all patients, according to the EUSTAR recommendations. All patients have given written informed consent for the recording and analysis of their medical data.

The entire cohort was used for survival analysis, while risk factor assessment was performed only on patients having at least 18 months of follow-up.

\section{STATISTICAL ANALYSIS}

Risk factors for death were analyzed on the entire cohort by logistic regression (LR) and also by Cox proportional hazards regression (Cox regression), both adjusted for age and sex. Kaplan-Meier survival curves were used to illustrate survival of different patient subsets.

Risk factors for an unfavorable disease outcome (excepting death) after the first 2 years ( \pm 6 months) from baseline were analyzed by age and sex-adjusted univariable LR; only patients who had a visit in the center at 2 years \pm 6 months since baseline were included in this analysis.

Risk factors for an unfavorable disease course, as assessed at the end of follow-up, were analyzed by age and sex-adjusted Cox regression. All patients having at last 18 months of follow-up were included in this analysis.

The following events were chosen to define an unfavorable disease course: increase in modified Rodnan score (mRSS) with at least $25 \%$ and 5 points (compared to baseline visit), decrease with at least $10 \%$ (compared to baseline) of predicted forced vital capacity (FVC) and predicted diffusing capacity of the lungs for carbon monoxide (DLCO), ejection fraction of the left ventricle (LVEF) lower than 50\% and presence of new digital ulcers (DUs).

Candidate risk factors were selected by clinical judgement among baseline parameters and included: male sex, age at baseline $>60$ years, disease duration at baseline $<3$ years, anti-centromere antibodies, an- ti-topoisomerase I antibodies, $\mathrm{mRSS}>14$, history of DUs, diffuse cutaneous subset, FVC $<70 \%$ of predicted, DLCO $<80 \%$ of predicted and proteinuria.

\section{RESULTS}

\section{Demographic data}

The entire study cohort comprised 137 patients (122 females (89.1\%) and 15 males (10.9\%), mean age $56.7 \pm 12.8$ years, disease duration $9.7 \pm 7.1$ years), of whom 117 patients $(85.4 \%)$ fulfilled the 1980 ACR classification criteria for SSc at the first visit.

\section{Duration of observation and risk factor analysis dataset}

96 patients had at least two visits, of whom 25 were considered lost to follow up (first visit prior to Jan 2014, without follow-up visit between Jan 2014 and March 2016). Of the remaining 71 patients, who had at least one follow-up visit after Jan 2014, 5 were excluded from the longitudinal analysis by LR (one died at $<6$ months after baseline, 4 did not have a follow-up visit at 2 years \pm 6 months after baseline). The remaining 66 patients constituted the analysis set for the risk factors analysis, with a median follow-up time of 4.4 years (range 2-19 years). Of them, 64\% had diffuse cutaneous SSc (dcSSc), 26\% had limited cutaneous SSc $(\mathrm{lcSSc})$ and 10\% were classified as overlap syndromes with SSc. Demographic and clinical data of all patients are shown in Table 1.

TABLE 1. Demographic data for the study cohort (137 patients). Data are means \pm standard deviations

\begin{tabular}{|l|l|}
\hline Age, years & $56.7 \pm 12.8$ \\
\hline Age at the onset of the Raynaud's phenomenon & $42.2 \pm 14.3$ \\
\hline Age at diagnosis & $46.9 \pm 13.0$ \\
\hline Disease duration since diagnosis, years & $9.7 \pm 7.1$ \\
\hline $\begin{array}{l}\text { Age at diagnosis for the diffuse cutaneous } \\
\text { subset ( } n=87)\end{array}$ & $51.6 \pm 13.3$ \\
\hline $\begin{array}{l}\text { Age at diagnosis for the limited cutaneous } \\
\text { subset ( } n=36)\end{array}$ & $48.2 \pm 11.6$ \\
\hline Age at diagnosis for men $(n=15)$ & $42.8 \pm 9.3$ \\
\hline Age at diagnosis for women $(n=122)$ & $47.4 \pm 13.4$ \\
\hline
\end{tabular}

\section{Survival analysis}

Nineteen deaths were reported (13.9\%), with 11 deaths directly related to SSc $(8.0 \%): 6$ by severe interstitial lung disease, 2 by pulmonary arterial hypertension, one by scleroderma renal crisis, one by severe denutrition and one sudden death. Non-SSc death causes were stroke ( 3 patients), malignancy (2 
patients), primary biliary cirrhosis, myocardial infarction and acute leukemia (one case each). Mean age at death was $57.9 \pm 11.4$ years, with mean disease duration at baseline of $6.7 \pm 5.2$ years. SSc-related death causes were associated with younger age at death, when compared to non-SSc related death causes. More data on SS-related and non-SSc related death are shown in Table 2.

TABLE 2. Non-SSc related versus SSc-related death causes $(n=19)$

\begin{tabular}{|l|c|c|c|}
\hline $\begin{array}{l}\text { Death } \\
\text { cause }\end{array}$ & $\begin{array}{c}\text { Age at } \\
\text { death } \\
\text { (mean } \pm \text { SD) }\end{array}$ & $\begin{array}{c}\text { Disease duration } \\
\text { (at death) } \\
\text { (mean } \pm \text { SD) }\end{array}$ & $\begin{array}{c}\text { Age at } \\
\text { diagnosis } \\
\text { (mean } \pm \text { SD) }\end{array}$ \\
\hline $\begin{array}{l}\text { Non-SSc } \\
\text { related } \\
\mathrm{N}=8\end{array}$ & $61.6 \pm 10.3$ & $8.4 \pm 7.5$ & $52.6 \pm 14.2$ \\
\hline $\begin{array}{l}\text { SSc-related } \\
\mathrm{N}=11\end{array}$ & $56.2 \pm 10.2$ & $5.5 \pm 2.3$ & $50.4 \pm 9.9$ \\
\hline
\end{tabular}

TABLE 3. Risk factors for death identified logistic regression $(L R)$ and Cox regression (both analyses were carried out with the candidate risk factor and age and sex as covariates)

\begin{tabular}{|c|c|c|c|}
\hline \multicolumn{4}{|c|}{ Risk factors for death ( $\mathrm{n}=137$ patients) } \\
\hline \multicolumn{4}{|l|}{ LR } \\
\hline & OR & $95 \% \mathrm{Cl}$ & $\mathrm{p}$ \\
\hline Male sex & 6.8 & $1.95-23.45$ & 0.003 \\
\hline $\mathrm{mRSS}>14$ & 6.9 & $2.22-21.32$ & 0.001 \\
\hline dcSSc & 4.8 & $1.27-17.93$ & 0.02 \\
\hline \multicolumn{4}{|c|}{ Cox Regression } \\
\hline & $\mathrm{HR}$ & $95 \% \mathrm{Cl}$ & $\mathrm{p}$ \\
\hline Male sex & 5.6 & $1.5-21.5$ & 0.012 \\
\hline Proteinuria & 7.7 & $1.4-43.3$ & 0.02 \\
\hline
\end{tabular}

TABLE 4. Risk factors for DLCO worsening ( $n=66$ patients): logistic regression (LR) and Cox regression (both analyses were carried out with the candidate risk factor and age and sex as covariates)

\begin{tabular}{|c|c|c|c|}
\hline \multicolumn{4}{|c|}{ Risk factors for DLCO worsening } \\
\hline \multicolumn{4}{|l|}{ LR } \\
\hline & OR & $95 \% \mathrm{Cl}$ & $p$ \\
\hline Male sex & 4.7 & $1.6-12.6$ & 0.004 \\
\hline \multicolumn{4}{|l|}{ Cox regression } \\
\hline & $\mathrm{HR}$ & $95 \% \mathrm{Cl}$ & $\mathrm{p}$ \\
\hline Male sex & 7.6 & $1.3-42.7$ & 0.023 \\
\hline dcSSc & 10.2 & $1.5-67.0$ & 0.016 \\
\hline \multicolumn{4}{|c|}{ Multivariate model LR } \\
\hline & OR & $95 \% \mathrm{Cl}$ & $\mathrm{p}$ \\
\hline Male sex & 9.1 & $1.2-70.4$ & 0.034 \\
\hline Age $<60$ years & 1.8 & $1.2-20.6$ & 0.635 \\
\hline dcSSc & 10.2 & $1.5-67.0$ & 0.016 \\
\hline
\end{tabular}

Risk factors for mortality identified by LR were: male sex, mRSS $>14$ at baseline and dcSSc, while Cox regression identified male sex and proteinuria as risk factors for death. Kaplan-Meier survival curves for patients with $\mathrm{dcSSc} / \mathrm{lcSSc}$ and respectively with $\mathrm{mRSS} \geq /<14$ at baseline are shown in Fig. 1 and 2.

\section{Disease evolution and prognostic factors}

During the entire follow-up, FVC improved in $30 \%$, was stable in $56 \%$ and worsened in $14 \%$, while DLCO improved in $7 \%$, was stable in $41 \%$ and worsened in $52 \%$ of the patients. mRSS improved in $25 \%$ of SSc patients, was stable in $71 \%$ patients, worsened in $5 \%$. New DUs occurred in $36 \%$ of the patients. $95 \%$ of patients who presented LVEF data at the last visit maintained LVEF $>50 \%$.

Risk factors for DLCO worsening identified by univariable LR were male sex and dcSSc, while unvariable Cox analysis identified only male sex. A multivariable prediction model for worsening of DLCO in the 2 years-analysis retained as significant risk factors male sex, age $<60$ years, and dcSSc.

The only risk factor identified for new DUs was the history of DUs at baseline (LR adjusted for age and sex: OR 4.2, 95\% CI 1.2-15.1).

We couldn't identify risk factors for the worsening of mRSS, FVC or LVEF because all analyses yielded statistically non-significant results.

\section{DISCUSSIONS}

Recent studies have shown that, over time, there has been a change in the pattern of death of patients with SSc, with a decrease in SSc-related causes (5). In our study, the proportion of SSc-related deaths was rather similar to the one of non-SSc related deaths: $8.0 \%$ versus $5.8 \%$. A recent study conducted on 987 patients concluded that pulmonary involvement is the main cause of deaths directly linked to SSc (5). SSc-related deaths in our patients were in almost $3 / 4$ of cases due to lung involvement ( 6 cases of ILD and 2 cases of pulmonary arterial hypertension), underlining the necessity of identifying predictive factors for lung function deterioration from the first visit. Male sex and dcSSc were identified as prognostic factors of DLCO deterioration.

Fifty-two percent of our patients presented deterioration of DLCO at the last visit, while for the other outcomes most of the patients presented a stable of favorable evolution (stable and improved $\mathrm{FVC}$, mRSS and no new DUs in 86\%, 96 and 64\%, respectively). This suggests that DLCO is more sensitive to change than FVC and reflects not only worsening of 


\section{Survival Functions}

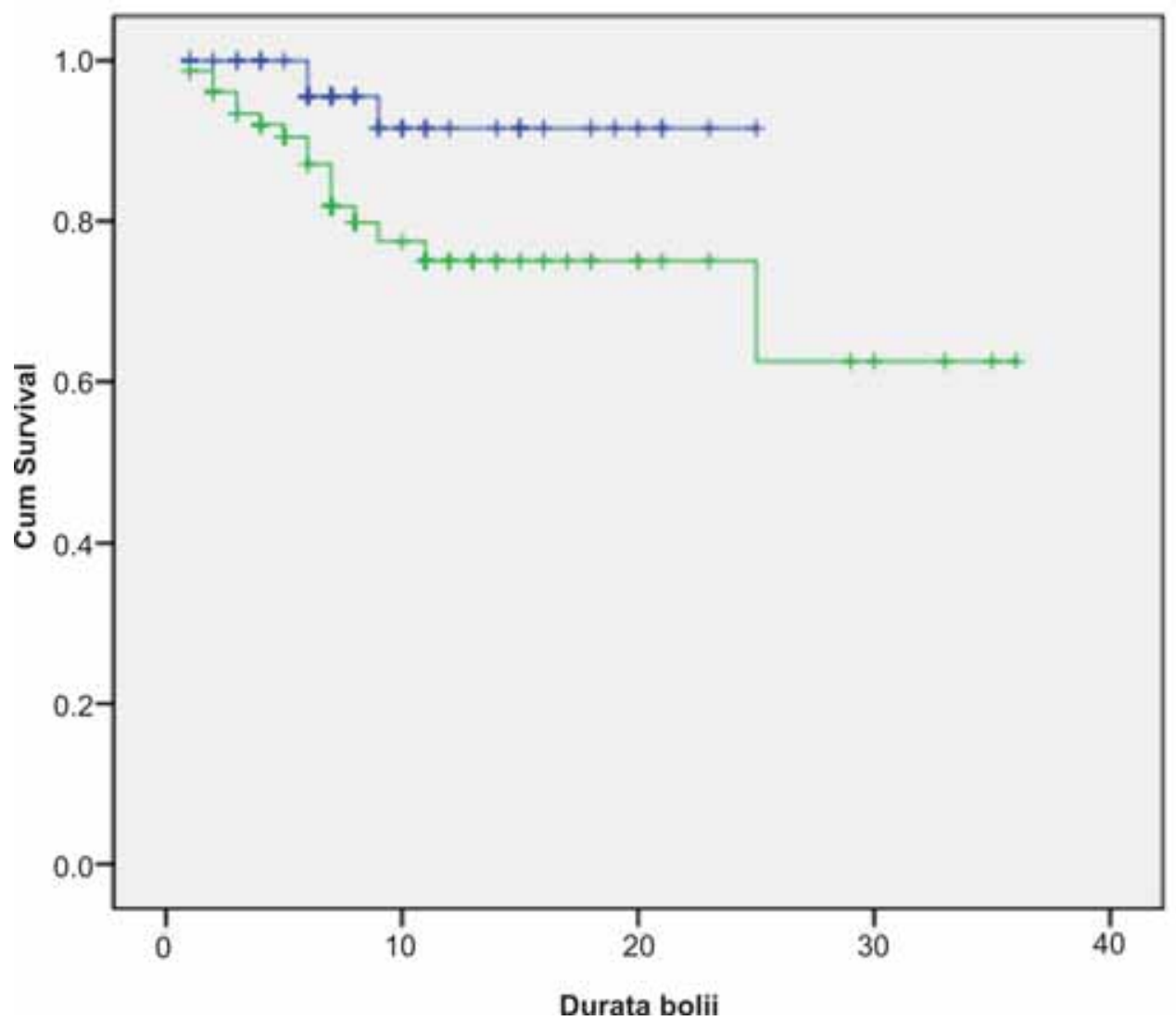

Subset

Tlimitat

$\rightarrow$ difuz

+ limitat-censored

I difuz-censored

FIGURE 1. Kaplan-Meier survival analysis for disease subset (Log Rank test $p=0.022)$

\section{Survival Functions}

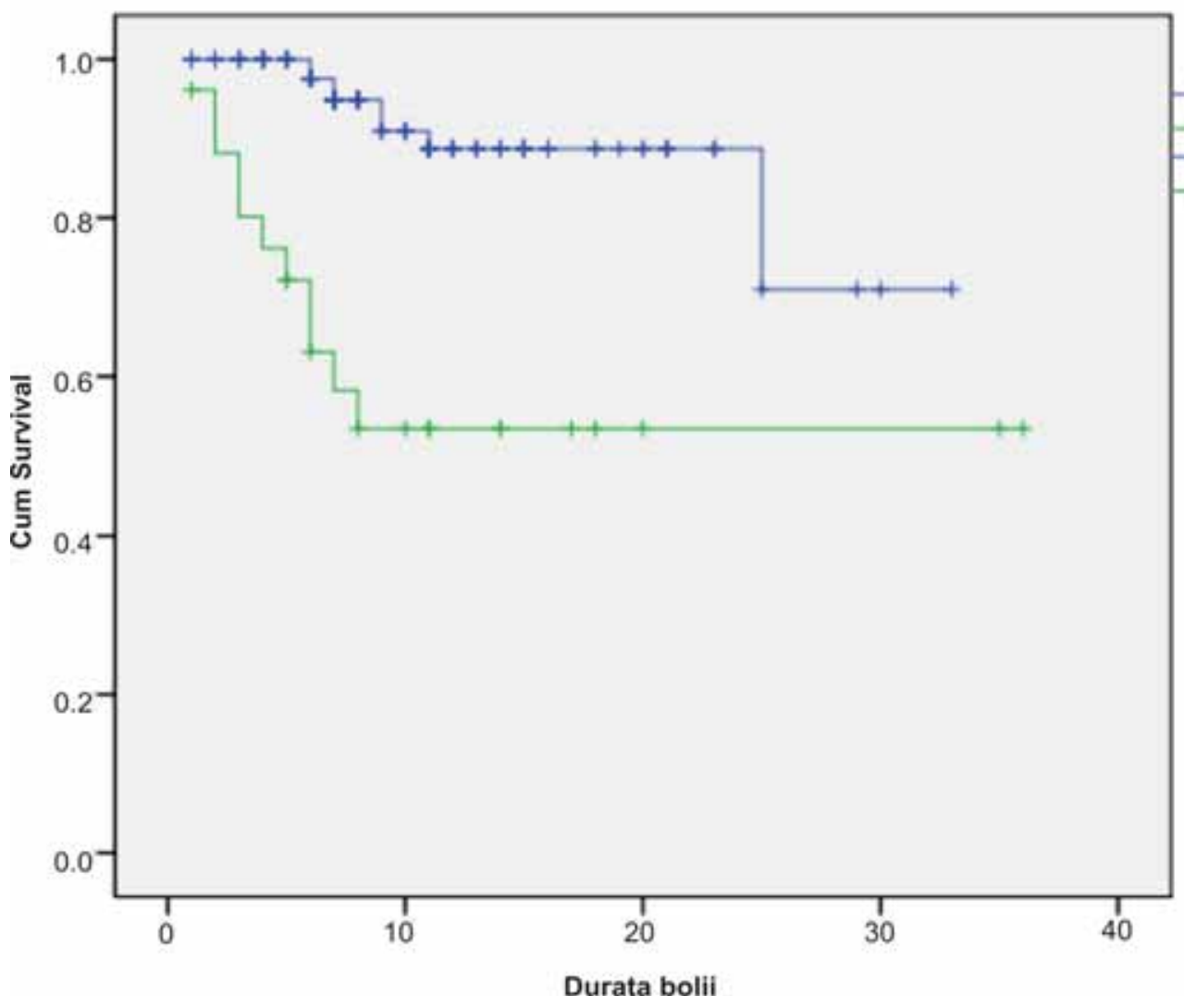

Scor Rodnan

mai mic de 14

mai mare de 14

- mai mic de 14-censored

mai mare de 14-censored 
SSc-related ILD, but also pulmonary hypertension and possibly other factors.

Another aim of this study was to construct a multivariate model for predicting disease worsening. This succeeded only for DLCO deterioration during the first 2 years of follow-up, using logistic regression, and included male sex, age $<60$ years and diffuse cutaneous SSc. When trying to determine a similar model by Cox regression, results were not statistically significant. Our results are similar to those of other studies on prognostic factors: diffuse cutaneous SSc as the only predictor of important lung involvement (6); DUs as the most important risk factor for occurrence of new DUs (7); increased erythrocyte sedimentation rate, anemia, low DLCO and proteinuria linked to high mortality (8).

Limitations of this study were deficiencies in data collecting for the follow up tests carried out in other facilities (with only $59 \%$ and $72 \%$ of patients having both at baseline and at the last visit pulmonary function tests, respectively echocardiography data), loss to follow up and the limited number of patients. Especially loss to follow up was a severe limitation, as 66 of the 137 patients in our cohort have no data on survival status after 2014. This was due mainly because many of these patients had been referred to our clinic for second opinion, but continued to attend another rheumatology facility. Because of assuming that all missing patients were survivors, our analysis of survival is rather optimistic.

\section{CONCLUSIONS}

SSc-related deaths were mainly due to lung involvement. Overall, prognostic factors for a worse disease course were male sex, diffuse cutaneous subset, disease duration $<3$ years and mRSS $>14$ at baseline. It is important to identify these prognostic factors at first visit of a SSc patient to provide proper disease management. For better management of SSc patients, the relationship between specific potential prognostic factors at first presentation and the disease outcome must be further studied in larger cohorts.

Conflict of interest: none declared Financial support: none declared

\section{REFERENCES}

1. Giacomelli R., Liakouli V., Berardicurti O. et al. Interstitial lung disease in Systemic sclerosis: current and future treatment, Rheum. Int. 2017, 853-863, 10.1007/s00296-016-3636-7.

2. Shreiner A.B., Murraz C., Denton C. et al., L Scleroderma Relat Disord. 2016; 1 (3):247-256, 10.5301/jsrd.5000214

3. Santosa A., Tan C.S., Teng G.G. et al. Lung and gastrointestinal complications are leading causes of death in SCORE, a multiethnic Singapore systemic sclerosis cohort, Scand J Rheumatol. 2016 Nov; 45(6):499-506. 27

4. Hao Y., Hudson M., Baron M. et al. Canadian Scleroderma Research Group; Australian Scleroderma Interest Group, Early Mortality in a Multinational Systemic Sclerosis Inception Cohort. Arthritis Rheumatol. 2016 Dec 28, 69: 1067-1077, 10.1002/ art. 40027

5. Rubio-Rivas M., Simeón-Aznar C.P., Velasco C. et al. Changes in the pattern of death of 987 patients with systemic sclerosis from
1990 to 2009 from the nationwide Spanish Scleroderma Registry (RESCLE), Clin Exp Rheumatol, 2017, 28229826.

6. Gilson M., Zerkak D., Wipff J. et al. Prognostic factors for cases, European Respiratory Journal 2010, 35: 112-117, 10.1183/09031936.00060209.

7. Mihai C., Landewé R., van der Heijde D. et al. Digital ulcers predict a worse disease in patients with systemic sclerosis, Ann Rheum Dis 2016, 75(4):681-6.

8. Hesselstrand R., Andréasson K., Wuttge D.M. et al. Increased serum COMP predicts mortality in SSc: results from a longitudinal study of interstitial lung disease, Rheumatology (Oxford) 2012, 51(5), 915-920. lung function in systemic sclerosis: prospective study of 105 